\title{
System Identification Using Laguerre Basis Functions
}

This paper was downloaded from TechRxiv (https://www.techrxiv.org).

\section{LICENSE}

CC BY 4.0

SUBMISSION DATE / POSTED DATE

28-01-2021 / 05-02-2021

CITATION

olivier, philip (2021): System Identification Using Laguerre Basis Functions. TechRxiv. Preprint. https://doi.org/10.36227/techrxiv.13656701.v1

$\mathrm{DOI}$

10.36227/techrxiv.13656701.v1 


\title{
SYSTEM IDENTIFICATION USING LAGUERRE BASIS FUNCTIONS
}

\author{
PHILIP D OLIVIER
}

\section{Contents}

1. Executive Summary. 1

2. Laguerre Basis Functions Background 3

2.1. Time domain vs z domain 3

2.2. Inner Products and Orthonormality 3

2.3. Pseudo Code for $\lambda_{k}(n) \quad 4$

3. The Product Property 5

3.1. A "Cauchy Product" for Laguerre basis functions. 5

4. System Identification: Third Order Approximations 6

5. Examples 9

6. Constraints 11

6.1. Matching Step Response Error. 12

6.2. Models without Direct Transmission 13

6.3. Matching a response to a given frequency 13

7. When is the model order correct? 13

8. discussion 14

9. conclusions 14

$\begin{array}{ll}\text { References } & 14\end{array}$

Abstract. This document describes how to use discrete time Laguerre basis functions, and their associated z-transforms, to construct a system identification process using experimentally determined Laguerre expansion coefficients of the input and output sequences. The process is derived using a new Product Property for the discrete time Laguerre basis functions. The system identification process is "linear in the parameters"; it does not require assumptions/knowledge of the poles locations of the system under test. An example is presented using data generated by a system that has appeared in the recent literature. The procedure naturally produces equations that can be used to determine if the chosen model order is correct or if its order needs to be increased. Constraints can easily be incorporated. Submitted to TechRxiv.org on Jan. 28, 2021.

\section{Executive Summary.}

The primary result of this effort is the development of a system identification procedure based on the experimentally determined Laguerre expansion coefficients of the input and output data. The Laguerre basis functions $\lambda_{k}[n] \leftrightarrow \Lambda_{k}(z)$ are 
orthonormal. Let $Y$ denote the column vector of the Laguerre coefficients of the system output, $U$ the column vector of the Laguerre coefficients of the system input, $a$ the Laguerre basis set parameter, $p$ the model parameters, and $M$ a "tall" rectangular matrix.

The system identification procedure presented here assumes that the system is excited by $\lambda_{0}(n)$ rather than by the more common impulse input.

The resulting system identification equations are summarized in a vector-matrix equation of the form

$$
M(Y, U, a) p=b
$$

The parameter vector, $p$, is determined by "solving" this equation, i.e. $p=M^{+} b$ where $M^{+}$is the pseudo inverse of $M$ so $p$ is the best least squares approximate solution to the over determined system.

The process has several useful features including

- it does not require prior knowledge of the poles of the system under test

- it is linear in the parameters

- it produces a check on whether the pre assumed model order is correct, analogous to the one found in Ho's algorithm.

The system identification equation is derived using a Product Property that is introduced in this paper for z-transform Laguerre basis functions. A similar Product Property was introduced for the Laplace transforms of continuous time Laguerre basis functions in 1995 by the author. It needs to be noted that what is referred to as Corollary 1 in this paper can be found in the 2018 paper of Manngard and Toivonen.

The product property that is used herein is of the form: Let $\Lambda_{k}(z)$ be the ztransform of a Laguerre basis function $\lambda_{k}(n)$ then

$$
\Lambda_{k}(z) \Lambda_{j}(z)=\Lambda_{0}(z) \Lambda_{k+j}(z)=\alpha \Lambda_{k+j}+\beta \Lambda_{k+j+1}
$$

where the $\alpha=\frac{1}{\sqrt{1-a^{2}}}$ and $\beta=\frac{-a}{\sqrt{1-a^{2}}}$.

This product property demonstrates the counter intuitive property that the product of two $\Lambda_{k}$ 's is the sum of two other $\Lambda_{k}$ 's. This has immediate implications for deconvolution and other applications in addition to the system identification problem considered here.

As mentioned before, the system identification procedure naturally produces a check on whether or not the pre assumed system model order is correct. ???????????????

$$
\text { ?????????????????????? }
$$

As lagniappe, it is noted that as a result of the product property, specifically that $\Lambda_{k} \Lambda_{j}=\Lambda_{0} \Lambda_{k+j}$, products of Laguerre series can be computed using a Cauchy Product like formula similar to the used to multiply power series.

The procedure is applied used to identify systems using data generated by a 5 th order system found in MT 2018. In MT 2018 a 2nd order system is identified. the systems identified using the approach described herein are found to be much superior when the errors in the step and impulse responses are compared to the analogous errors using the 2nd order system identified in MT 2018. 


\section{Laguerre Basis Functions Background}

. There are continuous time as well as discrete time Laguerre basis functions. These functions are orthornormal functions that $\operatorname{span} L_{2}$ and $l_{2}$ respectively. They have been used in systems and control engineering for many years see

- Peter S.C. Heuberger, Paul M.J. Van den Hof, Bo Wahlberg, (Eds.) Modelling and Identification with Rational Orthogonal Basis Functions, SpringerVerlag Lindon, 2005.

- P D Olivier, Approximate System Inverses, Electronic Letters, 1995, Vol. 31, No 23, pp 2050-2051

- Philip D Olivier, System Identification Using Laguerre Functions: Simple Examples, 29th Southeastern Symposium on System Theory, March 1997, Cookeville, TN pp 457-459.

- Philip D Olivier, Kevin Barnett, Signal Estimation via Laguerre Series, 42nd Southeastern Symposium on system Theory, Tyler TX March 7-9, 2010, pp 298-301

- M. Manngard, H.T. Toivonen, IFAC PapersOnLine 51-15 (2018) 72-77, doi: 10.1016/j.ifacol.2018.09.093.

Heuberger, et al (2005) contains a comprehensive summary of standard results and applications of discrete time Laguerre Basis Functions. Its chapters, and the references therein, are the most complete (as of 2005) set of references this author is aware of. Much of subsections 2.1 and 2.2 can be found in its pages. Olivier (1995) is the first use of a Product Property that the author is aware of. Olivier (1997) uses the product property for s-domain Laguerre functions to solve a continuous time system identification problem. In Olivier and Barnett (2010) the product property is used to identify signals and the resulting verification on the model order similar to that in Ho's algorithm was noted. Most recently, Manngard and Toivonen (2018) mix discrete time Laguerre orthonormal basis function theory and Ho's approach to system identification. They come close to presenting the full Product Property.

2.1. Time domain vs $\mathbf{z}$ domain. For $0 \leq k<\infty, a \neq 0,-1<a<1$ the Laguerre Basis Functions are defined by the following z-transform pairs $\lambda_{k}(n) \leftrightarrow \Lambda_{k}(z)$ where

$$
\begin{gathered}
\Lambda_{k}(z)=\sqrt{1-a^{2}} \frac{z(a z-1)^{k}}{(z-a)^{k+1}} \\
\lambda_{k}(n)=\sqrt{1-a^{2}} a^{n}(-1)^{k} \sum_{j=0}^{k}(-1)^{k+j}\left(\begin{array}{c}
k \\
j
\end{array}\right)\left(\begin{array}{c}
n+k-j \\
k
\end{array}\right) a^{k-2 j}
\end{gathered}
$$

These z-tansforms are bi-proper (numerator and denominator have same highest power of $\mathrm{z}$ ). This means that transfer functions expressed in terms of these Laguerre functions will probably include direct transmission (i.e. unless the parameters are chosen to minimize direct transmission).

2.2. Inner Products and Orthonormality. Laguerre basis functions are particularly useful in approximation/modeling situations because hey are orthonormal with 
respect to the standard inner products

$$
\begin{gathered}
<\lambda_{k}(n), \lambda_{j}(n)>=\sum_{n=0}^{\infty} \lambda_{k}(n) \lambda_{j}(n)=\delta_{k, j} \\
<\lambda_{k}(n), \lambda_{j}(n)>=<\Lambda_{k}(z), \Lambda_{j}(z)>=\frac{1}{2 \pi j} \oint_{|z|=1} \Lambda_{k}(z) \Lambda_{j}\left(\frac{1}{z}\right) \frac{d z}{z}
\end{gathered}
$$

where

$$
<\Lambda_{k}(z), \Lambda_{j}(z)>=\sum \text { residues }_{|z|<1}\left(\frac{\Lambda_{k}(z) \Lambda_{j}(1 / z)}{z}\right)
$$

2.3. Pseudo Code for $\lambda_{k}(n)$. This section modifies the expression for $\lambda_{k}(n)$ so it can be coded conveniently in numerical applications. Begin by replacing the binomial coefficients in

$$
\begin{array}{rlr}
\lambda_{k}(n) & = & \sqrt{1-a^{2}} a^{n}(-1)^{k} \sum_{j=0}^{k}(-1)^{k+j}\left(\begin{array}{c}
k \\
j
\end{array}\right)\left(\begin{array}{c}
n+k-j \\
k
\end{array}\right) a^{k-2 j} \\
& = & \sqrt{1-a^{2}} a^{n}(-1)^{k} \sum_{j=0}^{k}(-1)^{k+j} a^{k-2 j} \frac{k !}{(k-j) ! k !} \frac{(n+k-j) !}{(n-j) ! k !} \\
& = & \sqrt{1-a^{2}} a^{n}(-1)^{k} \sum_{j=0}^{k}(-1)^{k+j} \frac{a^{k-2 j}}{(k-j) ! j !} \frac{(n+k-j) !}{(n-j) !} \\
& = & \sqrt{1-a^{2}} a^{n}(-1)^{k} \sum_{j=0}^{k}(-1)^{k+j} \frac{a^{k-2 j}}{(k-j) ! j !}(n+k-j)(n+(k-1)-j) \ldots(n+1-j) \\
& = & \sqrt{1-a^{2}} a^{n}(-1)^{k} \sum_{j=0}^{k}(-1)^{k+j} \frac{a^{k-2 j}}{(k-j) ! j !} \prod_{q=1}^{k}(n+q-j)
\end{array}
$$

This last expression can be parsed into the easier to code $\lambda_{k}(n)=\sqrt{1-a^{2}} a^{n} P_{k}(n)$ where

$$
\begin{aligned}
& p_{k j}=\prod_{q=1}^{k}(n+q-j), \\
& P_{j k}(n)=(-1)^{k+j} \frac{a^{k-2 j}}{(k-j) ! j !} p_{k, j}(n), \text { and } \\
& P_{k}(n)=\sum_{j=0}^{k} P_{k j}(n) .
\end{aligned}
$$

\subsubsection{Pseudo code. Given $k$}

$\mathrm{P}=\operatorname{zeros}(\operatorname{size}(0: \mathrm{n}))$

for $\mathrm{j}=0: \mathrm{k}$

$$
\begin{aligned}
& \quad \mathrm{pkj}=1 ; \text { for } \mathrm{q}=1 \text { to } \mathrm{k} ; \mathrm{pkj}=\mathrm{pkj}(\mathrm{n}+\mathrm{q}-\mathrm{j}) \text {; end } \\
& \quad \mathrm{Pkj}=(-1)^{k+j}\left[a^{k-2 j} /((k-j) ! j !)\right] \mathrm{pkj} \\
& \quad \mathrm{P}=\mathrm{P}+\mathrm{Pkj} \\
& \text { end } \\
& \lambda_{k}(n)=\sqrt{1-a^{2}} a^{n}(-1)^{k} \mathrm{P}(n)
\end{aligned}
$$

end

The Matlab code based on the above pseudo code produced the following discrete time Laguerre functions for $a=0.5$ where $\theta(n)$ is the unit step function.

$$
\begin{aligned}
& \lambda_{0}(n)=[\sqrt{3} / 2](1 / 2)^{n} \\
& \text { [1] } \theta(n) \\
& \lambda_{1}(n)=[\sqrt{3} / 2](1 / 2)^{n} \quad[-1.5 n+.5] \quad \theta(n) \\
& \lambda_{2}(n)=[\sqrt{3} / 2](1 / 2)^{n} \quad\left[1.125 n^{2}-2.625 n+.25\right] \quad \theta(n) \\
& \lambda_{3}(n)=[\sqrt{3} / 2](1 / 2)^{n} \quad\left[-.5625 n^{3}-+.375 n^{2}-3.9375 n+.125\right] \quad \theta(n)
\end{aligned}
$$

Matlab was used to verify that they are each normal and that they are mutually orthogonal. 


\section{The Product Property}

A series of results are presented concerning products of z-domain Laguerre Basis Functions. It is common knowledge that products of z-domain functions correspond to convolutions of their inverse transforms in the time domain. The Product Property shows that for the Laguerre functions the product of z-domain basis functions are also sums of other z-domain basis functions. This Product Property is the primary new contribution of this work and forms the basis of less fundamental, though more applicable, contributions. These new contributions are contained in Theorem 1 regarding the Product Property for discrete time Laguerre functions. The author proved a similar result for continuous time Laguerre basis functions in [Olivier, 1995] and used it to obtain approximate system inverses. It needs to be acknowledged that Manngard and Toivonen (2018) proved a result equivalent to Corollary 1 presented below. As of 2018, the rest of the results in this section, and all of the developments in the next section, appear to be unknown.

Lemma 1 (Product Lemma). $\Lambda_{k}(z) \Lambda_{j}(z)=\Lambda_{0}(z) \Lambda_{k+j}(z)$

Proof.

$$
\Lambda_{k} \Lambda_{j}=\left(1-a^{2}\right) z^{2} \frac{(a z-1)^{k}(a z-1)^{j}}{(z-a)^{k+1}(z-a)^{j+1}}=\left(1-a^{2}\right) z^{2} \frac{(a z-1)^{k+j}}{(z-a)^{k+j+2}}=\Lambda_{0} \Lambda_{k+j}
$$

Theorem 1 (Product Property). $\Lambda_{k}(z) \Lambda_{j}(z)=\frac{\Lambda_{k+j}-a \Lambda_{k+j+1}}{\sqrt{1-a^{2}}}$

Proof.

$$
\begin{gathered}
\frac{\Lambda_{k+j}-a \Lambda_{k+j+1}}{\sqrt{1-a^{2}}}=\frac{z(a z-1)^{k+j}}{(z-a)^{k+j+1}}-\frac{z a(a z-1)^{k+j+1}}{(z-1)^{k+j+2}} \\
=\frac{z(a z-1)^{k+j}}{(z-a)^{k+j+2}}((z-a)-a(a z-1))=\frac{z^{2}(a z-1)^{k+j}}{(z-a)^{k+j+2}}\left(1-a^{2}\right)=\Lambda_{k} \Lambda_{j}
\end{gathered}
$$

Corollary 1. Corollary $\Lambda_{0} \Lambda_{k}=\frac{\Lambda_{k}-a \Lambda_{k+1}}{\sqrt{1-a^{2}}}$

3.1. A "Cauchy Product" for Laguerre basis functions. The Product Lemma (i.e. Lemma 1) shows that $\Lambda_{k} \Lambda_{j}=\Lambda_{0} \Lambda_{k+j}$ which is very similar to the property of polynomials $x^{k} x^{j}=x^{0} x^{k+j}$. This suggests that the product of Laguerre expansions in the frequency domain can be computed using an analog to the Cauchy Product familiar from the product of two polynomials:

$\left(\sum_{k=0}^{n} a_{k} x^{k}\right)\left(\sum_{j=0}^{m} b_{j} x^{j}\right)=\sum_{k=0}^{n}\left(\sum_{q=0}^{k} a_{q} b_{k-q}\right) x^{k}$

On the left, replace $x^{k}$ with $\Lambda_{k}, x^{j}$ with $\Lambda_{j}$ and on the right replace $x^{k}=x^{0} x^{k}$ with $\Lambda_{0} \Lambda_{k}$ yields

$\left(\sum_{k=0}^{n} a_{k} \Lambda_{k}\right)\left(\sum_{j=0}^{m} b_{j} \Lambda_{j}\right)=\sum_{k=0}^{n}\left(\sum_{q=0}^{k} a_{q} b_{k-q}\right) \Lambda_{0} \Lambda_{k}$

This similarity becomes more obvious below when the product of two expansions of Laguerre basis functions is computed explicitly. The rigorous proof follows, mutatis mutandis, of the proof of the Cauchy Product for polynomials, i.e. mix the summands, choose an appropriate change of variable of summation, and the result follows. 


\section{System Identification: Third Order Approximations}

System Identification begins with using the measured input-output data sequences $u(n)$ and $y(n)$ to generate numerical Laguerre expansions. This section provides a detailed discussion for $3^{\text {rd }}$ order systems. Modification for higher or lower order systems is straight forward.

$$
\begin{gathered}
u_{k}=<u(n), \lambda_{k}(n)>\quad \hat{U}=\sum_{k=0}^{\infty} u_{k} \Lambda_{k}(z) \approx \sum_{k=0}^{N} u_{k} \Lambda_{k}(z)= \\
y_{k}=<y(n), \lambda_{k}(n)>\quad \hat{Y}=\sum_{k=0}^{\infty} y_{k} \Lambda_{k}(z) \approx \sum_{k=0}^{N} y_{k} \Lambda_{k}(z)
\end{gathered}
$$

The unknown model is assumed to have a finite order (here, 3rd order, i.e. $\mathrm{N}=$ 3) model of the form

$$
\frac{\hat{Y}}{\hat{U}}=\hat{G}(z)=\frac{n_{0} \Lambda_{0}+n_{1} \Lambda_{1}+n_{2} \Lambda_{2}+n_{3} \Lambda_{3}}{d_{0} \Lambda_{0}+d_{1} \Lambda_{1}+d_{2} \Lambda_{2}+d_{3} \Lambda_{3}}=\frac{\sum_{k=0}^{N} n_{k} \Lambda_{k}}{\sum_{k=0}^{N} d_{k} \Lambda_{k}}
$$

For concreteness, the rational function equivalent is

$$
\hat{G}(z)=\frac{n_{0}(z-a)^{3}+n_{1}(a z-1)(z-a)^{2}+n_{2}(a z-1)^{2}(z-a)+n_{3}(a z-1)^{3}}{d_{0}(z-a)^{3}+d_{1}(a z-1)(z-a)^{2}+d_{2}(a z-1)^{2}(z-a)+d_{3}(a x-1)^{3}}
$$

It is conventional to normalize transfer functions so that the denominator is monic (i.e. highest power of $\mathrm{z}$ has coefficient 1 ), adopting this convention the result is constrained so that $d_{0}+a d_{1}+a^{2} d_{2}+a^{3} d_{3}=1$ for our example. In general the constraint is

$$
\sum_{k=0}^{N} a^{k} d_{k}=1
$$

The derivation of the system ID equations begins by clearing fractions to get (using $\mathrm{N}=2$, the pattern will emerge)

$$
\begin{aligned}
& \left(y_{0} \Lambda_{0}+y_{1} \Lambda_{1}+y_{2} \Lambda_{2}+y_{3} \Lambda_{3}+y_{4} \Lambda_{4}+y_{5} \Lambda_{5}+\ldots\right)\left(d_{0} \Lambda_{0}+d_{1} \Lambda_{1}+d_{2} \Lambda_{2}\right) \\
= & \left(u_{0} \Lambda_{0}+u_{1} \Lambda_{1}+u_{2} \Lambda_{2}+u_{3} \Lambda_{3}+u_{4} \Lambda_{4}+u_{5} \Lambda_{5}+\ldots\right)\left(n_{0} \Lambda_{0}+n_{1} \Lambda_{1}+n_{2} \Lambda_{2}\right)
\end{aligned}
$$

The products will be expanded and coefficients of $\Lambda_{k} \Lambda_{j}=\Lambda_{0} \Lambda_{k+j}$ are collected. Focusing on the left side product first.

The unprocessed system Identification equations are

$$
\sum_{k=0}^{\infty} y_{k} \Lambda_{k}\left(\sum_{p=0}^{N} d_{p} \Lambda_{p}\right)=\sum_{k=0}^{\infty} u_{k} \Lambda_{k} \sum_{p=0}^{N} n_{p} \Lambda_{p}
$$


The product of sums on the left will be expanded and simplified using the Product Property corollary.

$$
\begin{gathered}
\left(\sum_{k=0}^{\infty} y_{k} \Lambda_{k}\right)\left(\sum_{p=0}^{2} d_{p} \Lambda_{p}\right)= \\
\left(y_{0} \Lambda_{0}+y_{1} \Lambda_{1}+y_{2} \Lambda_{2}+y_{3} \Lambda_{3}+y_{4} \Lambda_{4}+y_{5} \Lambda_{5}+\ldots\right)\left(d_{0} \Lambda_{0}+d_{1} \Lambda_{1}+d_{1} \Lambda_{1}\right)= \\
y_{0} d_{0} \Lambda_{0} \Lambda_{0}+y_{0} d_{1} \Lambda_{0} \Lambda_{1}+y_{0} d_{2} \Lambda_{0} \Lambda_{2} \\
+y_{1} d_{0} \Lambda_{0} \Lambda_{1}+y_{1} d_{1} \Lambda_{1} \Lambda_{1}+y_{1} d_{2} \Lambda_{1} \Lambda_{2} \\
+y_{2} d_{0} \Lambda_{0} \Lambda_{2}+y_{2} d_{1} \Lambda_{1} \Lambda_{2}+y_{2} d_{2} \Lambda_{2} \Lambda_{2} \\
+y_{3} d_{0} \Lambda_{0} \Lambda_{3}+y_{3} d_{1} \Lambda_{1} \Lambda_{3}+y_{3} d_{2} \Lambda_{2} \Lambda_{3} \\
+y_{4} d_{0} \Lambda_{0} \Lambda_{4}+y_{3} d_{1} \Lambda_{1} \Lambda_{4}+y_{4} d_{2} \Lambda_{2} \Lambda_{4} \\
+y_{5} d_{0} \Lambda_{0} \Lambda_{5}+y_{5} d_{1} \Lambda_{1} \Lambda_{5}+y_{5} d_{2} \Lambda_{2} \Lambda_{5} \\
+\ldots
\end{gathered}
$$

Collecting coefficients of $\Lambda_{0} \Lambda_{k}$ (see similarity with products of polynomials here)

$$
\begin{aligned}
\left(\sum_{k=0}^{\infty} y_{k} \Lambda_{k}\right)\left(\sum_{p=0}^{2} d_{p} \Lambda_{p}\right)= & y_{0} d_{0} \Lambda_{0} \Lambda_{0}+\left(y_{1} d_{0}+y_{0} d_{1}\right) \Lambda_{0} \Lambda_{1} \\
& +\left(y_{2} d_{0}+y_{1} d_{1}+y_{0} d_{2}\right) \Lambda_{0} \Lambda_{2} \\
& +\left(y_{3} d_{0}+y_{2} d_{1}+y_{1} d_{2}\right) \Lambda_{0} \Lambda_{3} \\
& +\left(y_{4} d_{0}+y_{3} d_{1}+y_{2} d_{2}\right) \Lambda_{0} \Lambda_{4} \\
& +\left(y_{5} d_{0}+y_{4} d_{1}+y_{4} d_{2}\right) \Lambda_{0} \Lambda_{5} \\
& +\ldots
\end{aligned}
$$

Similarly

$$
\begin{aligned}
\left(\sum_{k=0}^{\infty} u_{k} \Lambda_{k}\right)\left(\sum_{p=0}^{2} n_{p} \Lambda_{p}\right)= & u_{0} n_{0} \Lambda_{0} \Lambda_{0}+\left(u_{1} n_{0}+y_{0} n_{1}\right) \Lambda_{0} \Lambda_{1} \\
& +\left(u_{2} n_{0}+u_{1} n_{1}+u_{0} n_{2}\right) \Lambda_{0} \Lambda_{2} \\
& +\left(u_{3} n_{0}+u_{2} n_{1}+u_{1} n_{2}\right) \Lambda_{0} \Lambda_{3} \\
& +\left(u_{4} n_{0}+u_{3} n_{1}+u_{2} n_{2}\right) \Lambda_{0} \Lambda_{4} \\
& +\left(u_{5} n_{0}+u_{4} n_{1}+u_{4} n_{2}\right) \Lambda_{0} \Lambda_{5} \\
& +\ldots
\end{aligned}
$$

Recalling the product property corollary $\Lambda_{0} \Lambda_{k}=\frac{1}{\sqrt{1-a^{2}}} \Lambda_{k}-\frac{a}{\sqrt{1-a^{2}}} \Lambda_{k+1}$. This specialized product property is used to express the product of sums expression as a sum of Laguerre basis functions yields

$$
\begin{gathered}
\left(\sum_{k=0}^{\infty} y_{k} \Lambda_{k}\right)\left(\sum_{p=0}^{2} d_{p} \Lambda_{p}\right)=y_{0} d_{0} \frac{\Lambda_{0}-a \Lambda_{1}}{\sqrt{1-a^{2}}}+\left(y_{1} d_{0}+y_{0} d_{1}\right) \frac{\Lambda_{1}-a \Lambda_{2}}{\sqrt{1-a^{2}}}+ \\
\left(y_{2} d_{0}+y_{1} d_{1}+y_{0} d_{2}\right) \frac{\Lambda_{2}-a \Lambda_{3}}{\sqrt{1-a^{2}}}+ \\
\left(y_{3} d_{0}+y_{2} d_{1}+y_{1} d_{2}\right) \frac{\Lambda_{3}-a \Lambda_{4}}{\sqrt{1-a^{2}}}+ \\
\left(y_{4} d_{0}+y_{3} d_{1}+y_{2} d_{2}\right) \frac{\Lambda_{4}-a \Lambda_{5}}{\sqrt{1-a^{2}}} \\
+\ldots
\end{gathered}
$$


Now collect the terms with $\Lambda_{k}$ and within them collect terms in $d_{i}$.

$$
\begin{gathered}
\sqrt{1-a^{2}}\left(\sum_{k=0}^{\infty} y_{k} \Lambda_{k}\right)\left(\sum_{p=0}^{2} d_{p} \Lambda_{p}\right) \\
=y_{0} a_{0} \Lambda_{0} \\
+\left(\left(y_{1}-a y_{0}\right) d_{0}+y_{0} d_{1}\right) \Lambda_{1} \\
+\left(\left(y_{2}-a y_{1}\right) d_{0}+\left(y_{1}-a y_{0}\right) d_{1}+y_{0} d_{2}\right) \Lambda_{2} \\
+\left(\left(y_{3}-a y_{2}\right) d_{0}+\left(y_{2}-a y_{1}\right) d_{1}+\left(y_{1}-a y_{0}\right) d_{2}\right) \Lambda_{3} \\
+\left(\left(y_{4}-a y_{3}\right) d_{0}+\left(y_{3}-a y_{2}\right) d_{1}+\left(y_{2}-a y_{1}\right) d_{2}\right) \Lambda_{4} \\
+\ldots
\end{gathered}
$$

Similarly

$$
\begin{gathered}
\sqrt{1-a^{2}}\left(\sum_{k=0}^{\infty} u_{k} \Lambda_{k}\right)\left(\sum_{p=0}^{2} n_{p} \Lambda_{p}\right) \\
=u_{0} n_{0} \Lambda_{0} \\
+\left(\left(u_{1}-a u_{0}\right) n_{0}+u_{0} n_{1}\right) \Lambda_{1} \\
+\left(\left(u_{2}-a u_{1}\right) n_{0}+\left(u_{1}-a u_{0}\right) n_{1}+u_{0} n_{2}\right) \Lambda_{2} \\
+\left(\left(u_{3}-a u_{2}\right) n_{0}+\left(u_{2}-a u_{1}\right) n_{1}+\left(u_{1}-a u_{0}\right) n_{2}\right) \Lambda_{3} \\
+\left(\left(u_{4}-a u_{3}\right) n_{0}+\left(u_{3}-a u_{2}\right) n_{1}+\left(u_{2}-a u_{1}\right) n_{2}\right) \Lambda_{4} \\
+\ldots
\end{gathered}
$$

Now the coefficients of $\Lambda_{k}$ can be equated from

$$
\begin{aligned}
& 0=\sum_{k=0}^{\infty} u_{k} \Lambda_{k} \sum_{p=0}^{2} n_{k} \Lambda_{p}-\sum_{k=0}^{\infty} y_{k} \Lambda_{k} \sum_{p=0}^{2} d_{k} \Lambda_{p} \\
& 0=\mathrm{u}_{0} n_{0}-\left[y_{0} d_{0}\right] \\
& 0=\left(\mathrm{u}_{1}-a u_{0}\right) n_{0}+u_{0} n_{1}-\left[\left(y_{1}-a y_{0}\right) d_{0}+y_{0} d_{1}\right] \\
& 0=\left(\mathrm{u}_{2}-a u_{1}\right) n_{0}+\left(u_{1}-a u_{0}\right) n_{1}+u_{0} n_{2}-\left[\left(y_{2}-a y_{1}\right) d_{0}+\left(y_{1}-y_{0}\right) d_{1}+y_{0} d_{2}\right] \\
& \text { for } k \geq 3 \\
& 0=\left(\mathrm{u}_{3}-a u_{2}\right) n_{0}+\left(u_{2}-a u_{1}\right) n_{1}+\left(u_{1}+a u_{0}\right) n_{2}-\left[\left(y_{3}-a y_{2}\right) d_{0}+\left(y_{2}-y_{1}\right) d_{1}+\left(y_{1}-y_{0}\right) d_{2}\right]
\end{aligned}
$$

These equations can be put in matrix form $0=-\hat{D}(\hat{Y}) d+\hat{N}(\hat{U}) n=M(\hat{U}, \hat{Y}) p$ where $\hat{Y}$ is the column vector of the $y_{k}$ 's, $\hat{U}$ is the column vector of the $u_{k}$ 's, $n$ is the column vector of the $n_{k}$ 's, $d$ is the column vector of the $d_{k}$ 's, $p$ is the column vector with $d$ stacked on top of $n$, and the matrices $\hat{N}$ and $\hat{D}$ are given by (there should be $N+1$ columns in each matrix)

$$
\hat{N}=\left[\begin{array}{ccc}
u_{0} & 0 & 0 \\
u_{1}-a u_{0} & u_{0} & 0 \\
u_{2}-a u_{1} & u_{1}-a u_{0} & u_{0} \\
u_{3}-a u_{2} & u_{2}-a u_{1} & u_{1}-a u_{0} \\
\vdots & \vdots & \vdots
\end{array}\right], \quad \hat{D}=\left[\begin{array}{ccc}
y_{0} & 0 & 0 \\
y_{1}-a y_{0} & y_{0} & 0 \\
y_{2}-a y_{1} & y_{1}-a y_{0} & y_{0} \\
y_{3}-a y_{2} & y_{2}-a y_{1} & y_{1}-a y_{0} \\
\vdots & \vdots & \vdots
\end{array}\right]
$$

As it stands now $p=0$ is a solution to $M p=0$ which is reasonable. The constraint discussed above needs to be added. Recall the constraint is $d_{0}+a d_{1}+a^{2} d_{2}+a^{3} d_{3}=1$. 
This constraint, as well as other linear constraints, can be expressed in matrix form

$$
p=\left[\begin{array}{l}
d_{0} \\
d_{1} \\
d_{2} \\
d_{3} \\
n_{0} \\
n_{1} \\
n_{2} \\
n_{3}
\end{array}\right]=\left[\begin{array}{l}
1 \\
0 \\
0 \\
0 \\
0 \\
0 \\
0 \\
0
\end{array}\right]+\left[\begin{array}{ccccccc}
-a & -a^{2} & -a^{3} & 0 & 0 & 0 & 0 \\
1 & 0 & 0 & 0 & 0 & 0 & 0 \\
0 & 1 & 0 & 0 & 0 & 0 & 0 \\
0 & 0 & 1 & 0 & 0 & 0 & 0 \\
0 & 0 & 0 & 1 & 0 & 0 & 0 \\
0 & 0 & 0 & 0 & 1 & 0 & 0 \\
0 & 0 & 0 & 0 & 0 & 1 & 0 \\
0 & 0 & 0 & 0 & 0 & 0 & 1
\end{array}\right]\left[\begin{array}{l}
d_{1} \\
d_{2} \\
d_{3} \\
n_{0} \\
n_{1} \\
n_{2} \\
n_{3}
\end{array}\right]=\hat{b}+C \hat{p}
$$

The resulting system identification equation can be put in the final form

$$
0=M(\hat{b}+C \hat{p}) \quad M C \hat{p}=-M \hat{b}
$$

The system identification procedure produces an infinite number equations because there are normally an infinite number of non-zero Laguerre coefficients of the output and/or input sequences, $y_{k}$, and $u_{k}$. These infinite number of equations need to be truncated in any actual application after their components become small enough.

Notice that each column in $N$ (or $D$ ) is a shifted version of the previous column with a zero added at the top; the number of columns of $N$ and $D$ is the same as the number of Laguerre basis functions used in the model; and, the columns of each matrix are independent of the model order.

The parameter vector is obtained by finding the best approximate solution to the over determined set of equations summarized in $M C \hat{p}=-M \hat{b}$. Using the pseudo inverse of $M C,(M C)^{+}$, producing the $\hat{p}$ that minimizes the error

$$
e=-M \hat{b}-M C \hat{p}
$$

in the least-squares sense.

Using only a finite number of the equations (i.e. truncating the equations) will produce an approximate solution. the accuracy of the solution is controlled by truncating the equations only after the components of the vectors/matrices are sufficiently close to zero.

\section{EXAMPLES}

The developed procedure will now be applied to identify systems from data generated by a known 5th order system found in MT 2018. The systems identified using the Laguerre based procedure will be compared to the 2nd order system identified in MT 2018.

to begin with the 5 th order system that will be used to generate the data is

$$
G(z)=\frac{.2530 z^{4}-.9724 z^{3}+1.4283 z^{2}-.9493 z .2410 .}{z^{5}-4.15 z^{4}+6.8831 z^{3}-5.6871+2.3333 z-.3787}
$$

This system has poles at $0.95 \pm j .20, .85 \pm j .10, .55$.

The 2nd order system identified in MT 2018 is

$$
H(z)=\frac{z^{2}-1.38 z+.4}{z^{2}-1.90 z+.91}
$$




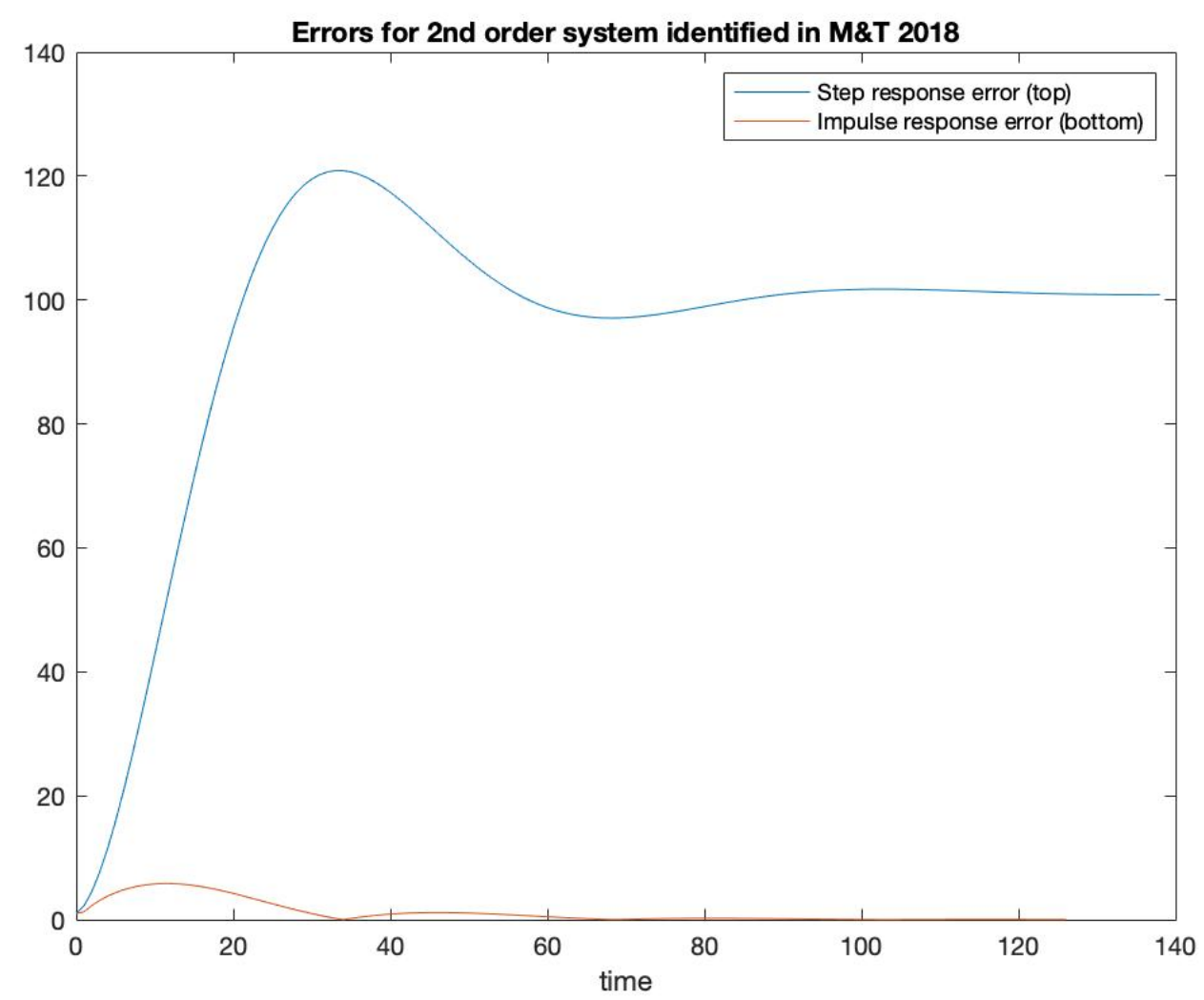

FiguRE 1. These plots compare the 2nd order system identified in MT 2018 to the system that generated the data. It shows the plots the error of the step response and the error of the impulse response.

The system identified in MT2018 is not particularly good as can be seen by looking at the errors in the step and impulse response presented in Figure 1.

The system expounded on in this paper identified the following two systems data generated from $G(z)$ with $\lambda_{0}(n)$ as input.

$$
G_{L 2}(z)=\frac{0.0439 z^{2}+0.1123 z-0.1481}{z^{2}-1.442 z-0.4516}
$$

This system has poles at 0.9822 and 0.4598 .

$$
G_{L 3}(z)=\frac{0.0012 z^{3}+0,2471 z^{2}-0.4701 z+0.2398}{z^{3}-2.212 z^{2}+1.635 z-0.4041}
$$

This system has poles at 0.7938 and $0.7090 \pm 0.0804 j$.

The efficacy of the Laguerre models is demonstrated by the reduction in the impulse response error and the step response error as compared to that of the Maangard and Toivenen model shown in the previous figure. The impulse errors

The step errors 


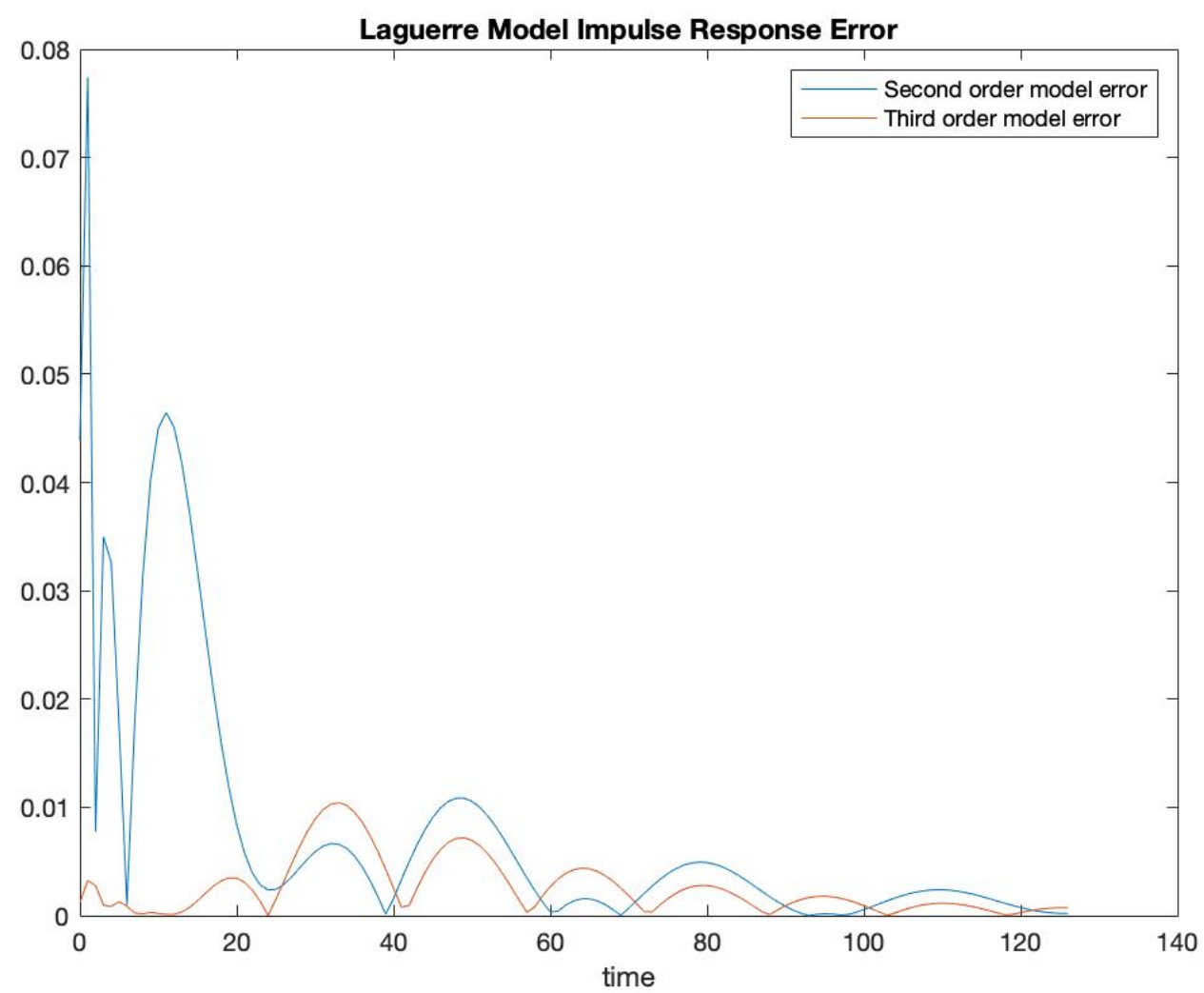

Figure 2. These plots present the error in impulse responses for the second and third order Laguerre models.

\section{Constraints}

The Laguerre system identification procedure required a constraint: the monic denominator constraint $p=d+C \hat{p}$ to get

$$
M C \hat{p}=\hat{b}
$$

which had non-trivial solutions. Other constraints can be included

A constraint can be either soft or hard. Soft constraints can be implemented by augmenting the identification equations with additional linear expressions, perhaps wighted, that nudge the model toward the desired feature expressed in the constraint. Implementation of hard constraints is the subject of this section.

Often a constraint can be defined by expressing the parameter vector, $p$ in terms of a subset of the parameter vector, $\tilde{p}$ and perhaps a constant $\beta$

$$
p=C \tilde{p}+\beta
$$

More than one constraint can be included in this equation.

The system identification problem then reduces to finding the least squares solution to the over determined set of equations

$$
M C \tilde{p}=\bar{y}-M \beta
$$




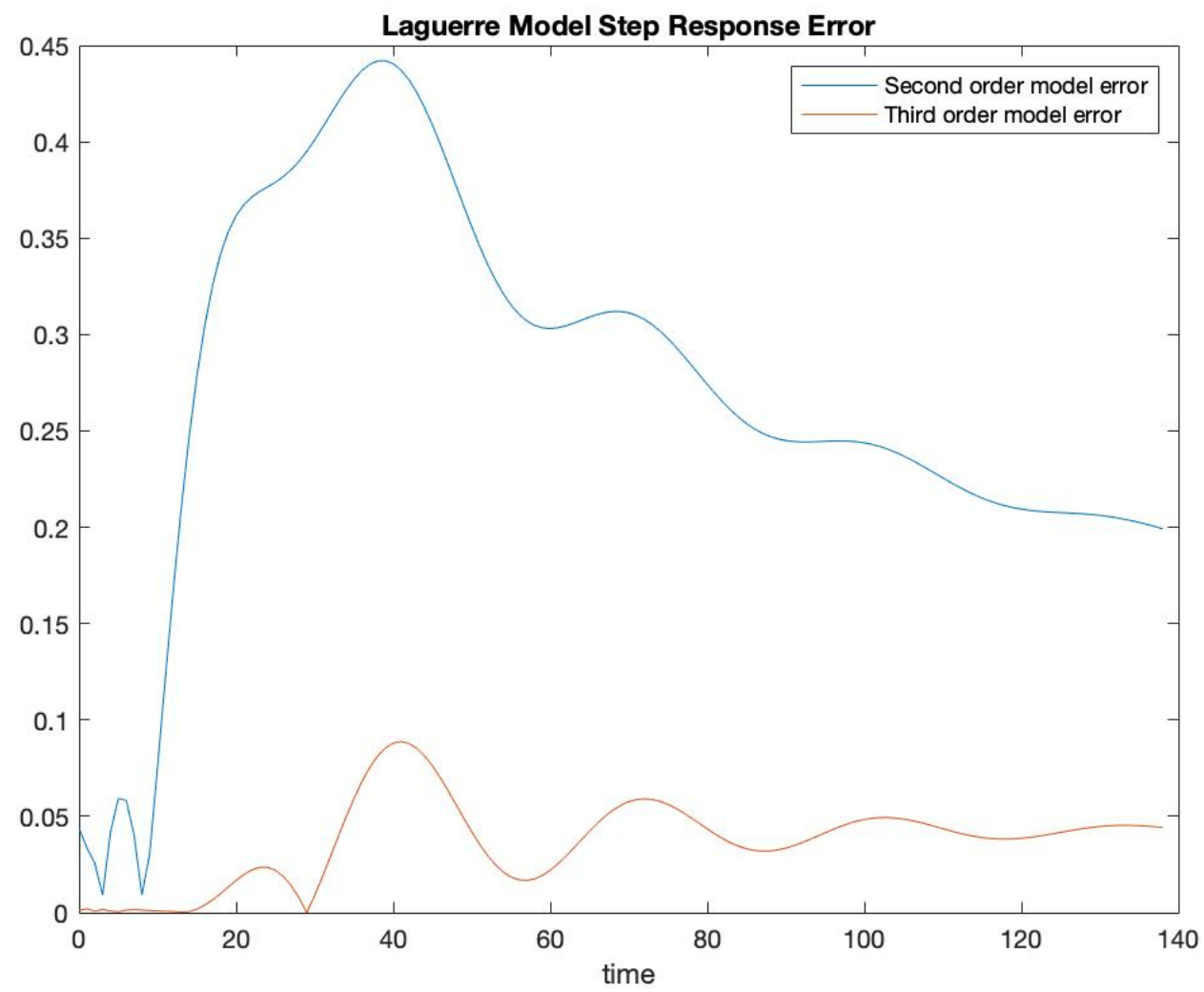

FiguRE 3. These plots present the error in step responses for the second and third order Laguerre models

Various constraints can easily be imposed on the model before the least-squares approximation is done provided those constraints can be stated as linear expressions in the unknown coefficients. Two such situations will demonstrate the point.

6.1. Matching Step Response Error. Recall the Final Value Theorem which, assuming both limits exist, says that $\lim _{n->\infty} f(n)=\lim _{z->1}(z-1) F(z)$. If experiments on the unit under test indicate that $y_{\text {step,ss }}=\lim _{n->\infty} y_{\text {step }}(n)=\lim _{z->1}(z-$ 1) $G(z) z /(z-1)=G(1)$. So to preserve this feature of the UUT in the identified model

$$
\sqrt{1-a^{2}} \sum_{k=0}^{N}(-1)^{k} a_{k}=F(1)\left((-a)^{N+1}+\sqrt{1-a^{2}} \sum_{k=0}^{N}(-1)^{k} b_{k}\right)
$$

Solving for $b_{N}$

$$
b_{N}=a^{N+1}+\frac{1}{\sqrt{1-a^{2}} F(1)} \sum_{k=0}^{N} a_{k}-\sum_{k=0}^{N-1}(-1)^{k} b_{k}
$$


6.2. Models without Direct Transmission. Model transfer functions that are bi proper have direct transmission, whereas model transfer functions that are strictly proper have no direct transmission. This is easy to see from the state-space model and its transfer function $x(n+1)=A x(n)+B u(n), y(n)=C x(n)+d u(n)<->$ $H(z)=C(z I-A)^{-1}+d$. When $d=0$ the transfer function is strictly proper and the system has no direct transmission.

The constraint equations comes from guaranteeing that numerator polynomial has lower order than the denominator polynomial. That is, the coefficient of $z^{n+1}$ must be zero. The numerator polynomial is

$$
N(z)=\sqrt{1-a^{2}} \sum_{k=0}^{N} a_{k} z(a z-1)^{k}(z-a)^{N-k}
$$

Setting the coefficient of $z^{N+1}$ to zero produces

$$
\sqrt{1-a^{2}} \sum_{k=0}^{N} a_{k} a^{k}=0
$$

Solving for $a_{N}$

$$
a_{N}=-\frac{1}{a^{N}} \sum_{k=0}^{N-1} a_{k} a^{k}
$$

6.3. Matching a response to a given frequency. It is often desired to either block a given frequency or to allow a given frequency to pass unaltered. To block a signal at frequency $\omega$, require $N\left(e^{j \omega}\right)=0$. To allow unaltered passage of this signal require $\frac{N\left(e^{j \omega}\right)}{D\left(e^{j \omega}\right)}=1$. Either case is expressible as a complex conjugate pair of linear equations in the numerator coefficients.

\section{WHEN IS THE MODEL ORDER CORRECT?}

To answer this question begin with unconstrained system identification equations

$$
D d=N n
$$

When the test input is $\lambda_{0}$, only the first few rows of $N$ have non zero entries. Aslo assume an exact model, i.e. the coefficients of $d$ are correct, the computation of the $y_{k}$ are perfect, the model order is correct and is $R$. Now choose a $k$ so all entries in the $k^{t h}$ row of $\mathrm{N}$ are zero.Then for any $r \geq k$. The $r^{t h}$ row of Equation(18) is

$$
\sum_{i=1}^{R}\left(y_{k+i}-a y_{k+i-1}\right) d_{i-1}=0
$$

Solving this for $y_{k+R}$

$$
y_{k+R}=\frac{a y_{k+R-1}+\sum_{i=1}^{R-1}\left(y_{k+i}-a y_{k+i-1}\right) d_{i-1}}{d_{R-1}}
$$


So consider creating a sequence of models of increasing order, $R=1, R=2, \ldots$ $R=R^{*}, \ldots$ At each step compute

$$
\sum_{i=1}^{R}\left(y_{k+i}-a y_{k+i-1}\right) d_{i-1}=\epsilon_{R}
$$

The "optimal" model order is $R^{*}$ provided $\epsilon_{R^{*}}=0$.

Now what happens for more realistic conditions? Say computations are imprecise, or the actual system is not finite dimensional, then the "optimal" model order is $R^{*}$ provided $\left|\epsilon_{R^{*}}\right|<<\left|\epsilon_{R^{*}-1}\right|$

The classic system identification method proposed by Ho, Kalman and Narendra that was based on impulse response provided a similar test for model order correctness.

\section{DISCUSSION}

\section{CONCLUSIONS}

\section{REFERENCES}

[1] Peter S. C. Heuberger, Paul M.J. Van den Hof, Bo Wahlberg, Eds., Modelling and Identification with Rational Orthogonal Basis Functions, Springer-Verlag, London, 2005.

[2] Manngard, M. Toivonen, H.T., IFAC PapersOnLine 51-15 (2018) 72-77, doi: 10.1016/j.ifacol.2018.09.093.

[3] Olivier, PD, Approximate System inverses, electronic Letters, 1995, Vol. 31, No 23 pp 20502051.

[4] Philip D Olivier, Kevin D Barnett, Signal Estimation via Laguerre Series, 42nd SouthEastern Symposium on system Theory, Tyler Texas, USA, March 7-9, 2010, pp. 298-301.

[5] Philip D Olivier, System Identification Using Laguerre Functions: Simple Examples, 29th Southeastern Symposium on Theory, March1997 Cookeville, TN pp 457-459.

[6] need this citation to Ho algorithm 